\title{
ON DISTORTION THEOREMS INVOLVING GENERALIZED FRACTIONAL CALCULUS OPERATORS*
}

\author{
R. K. RAINA AND MAMTA BOLIA
}

\begin{abstract}
This paper gives new classes of distortion inequalities for various subclasses of analytic and univalent functions. The results presented involve certain generalized fractional integrals of functions belonging to the general classes $J_{\delta}(n)$ and $L_{\delta}(n)$. Some useful deductions of our main results are also pointed out.
\end{abstract}

\section{Introduction}

The study of generalized operators of fractional integrals (or derivatives) having as kernels various types of special functions including the Fox's H-function have been receiving keen attention presently. For recent works on the subject, one may refer to [2], [3], [6] and [9].

The theory of fractional calculus operators have also been applied to univalent function theory, and recently Srivastava, Saigo and Owa [12] used the Saigo's fractional calculus operators [8] to prove certain classes of distortion theorems for various subclasses of analytic and univalent functions.

The present paper is devoted to the investigation of new classes of distortion inequalities for substantially more generalized forms of fractional integral operators (involving Fox's H-function) belonging to the general classes of analytic and univalent functions $J_{\delta}(n)$ and $L_{\delta}(n)$ (defined below by (2.5) and (2.6), respectively). As pointed out in [2] and [6] (see also [9, pp.141-145]) the fractional integral operator with Fox's H-function in the kernel includes various fractional integral operators, like the Riemann-Liouville fractional integral operators, the Kober fractional integrals, Erdélyi fractional integrals, the Love fractional integrals and also Saigo fractional integrals. Therefore, the results

Received January 17, 1995

1991 Mathematics Subject Classification. 26A33, 30C45, 30C99, 33C40.

Key words and phrases. Distortion inequalities, fractional integrals, Fox's H-function, Univalent functions.

* Dedicated to the memory of Late Dr. Ram Kumar, DSC. (formerly Professor and Head, Dept. of Mathematics, University of Udaipur, Udaipur (India)). 
presented in this paper possess wider applicabilities. We give in the concluding section some examples illustrating the useful deductions that emerge from our main results.

The set of nonnegative integers is denoted by $N$ and $N_{0}=N U\{0\} \cdot R$ means the field of real numbers, $C$ is the complex number field, and $R_{+}=(0, \infty)$.

Throughout this paper $\left(a_{m}\right)$ stands for the $m$-parameters:

$a_{1}, \ldots, a_{m} \quad(m \in N)$; and the symbolic form $\left(a_{j}, \alpha_{j}\right)_{1, m}$ abbreviates the set of parameters $\left(a_{1}, \alpha_{1}\right), \ldots,\left(a_{m}, \alpha_{m}\right), \quad m \in N$.

Also, $(\lambda)_{n}=\frac{\Gamma(\lambda+n)}{\Gamma(\lambda)}$ denotes the usual Pochhammer symbol.

\section{Preliminaries and definitions}

Let $T(n)$ denote the class of functions of the form

$$
f(z)=z+\sum_{j=n+1}^{\infty} a_{j} z^{j} \quad(n \in N),
$$

which are analytic in the unit disk $U=\{z:|z|<1\}$. Further, let $S(n)$ denote the class of all functions in $T(n)$ which are univalent in the unit disk $U$. Then a function $f(z) \in S(n)$ is said to be in the subclass $S_{\delta}(n)$ if and only if

$$
R e\left\{\frac{z f^{\prime}(z)}{f(z)}\right\}>\delta \quad(z \in U),
$$

for some $\delta(0 \leq \delta<1)$. Also, a function $f(z) \in S(n)$ is said to be in the subclass $K_{\delta}(n)$ if and only if

$$
\operatorname{Re}\left\{1+\frac{z f^{\prime \prime}(z)}{f^{\prime}(z)}\right\}>\delta \quad(z \in U),
$$

for some $\delta(0 \leq \delta<1)$.

We note that $f(z) \in K_{\delta}(n)$ if and only if $z f^{\prime}(z) \in S_{\delta}(n)$, and that

$$
S_{\delta}(n) \subseteq S_{0}(n), \quad K_{\delta}(n) \subseteq K_{0}(n), \quad \text { and } \quad K_{\delta}(n) \subseteq S_{\delta}(n) \quad \text { for } \quad 0 \leq \delta<1 .
$$

The classes $S_{\delta}(n)$ and $K_{\delta}(n)$ were studied recently by Srivastava, Owa and Chatterjea [11].

It may be noted that for $n=1, S_{\delta}(1)$ and $K_{\delta}(1)$ precisely turn out to be the classes $S^{*}(\delta)$ and $K(\delta)$, respectively, which were introduced earlier by Robertson [7].

Also, let $J(n)$ be the subclass of $S(n)$ consisting of functions of the form

$$
f(z)=z-\sum_{j=n+1}^{\infty} a_{j} z^{j} \quad\left(a_{j} \geq 0\right) .
$$

We denote by $J_{\delta}(n)$ and $L_{\delta}(n)$ the classes obtained by taking intersections respectively of the classes $S_{\delta}(n)$ and $K_{\delta}(n)$ with $J(n)$; i.e.

$$
J_{\delta}(n)=S_{\delta}(n) \cap J(n) \quad(0 \leq \delta<1 ; n \in N),
$$


and

$$
L_{\delta}(n)=K_{\delta}(n) \cap J(n) \quad(0 \leq \delta<1 ; n \in N) .
$$

The classes $J_{\delta}(n)$ and $L_{\delta}(n)$ were considered earlier by Chatterjea [1]. In particular, $J_{\delta}(1)$ and $L_{\delta}(1)$ correspond to the classes $J^{*}(\delta)$ and $L(\delta)$, respectively, introduced by Silverman [10].

We shall be concerned in this paper with the fractional integral operators involving the well-known Fox's H-function introduced recently in [3] (see also [2] and [9]).

Definition. Let $m \in N, \beta_{k} \in R_{+}$and $\gamma_{k}, \delta_{k} \in C, \forall k=1, \ldots, m$; with $\sum_{k=1}^{m} \operatorname{Re}\left(\delta_{k}\right)>0$. Then the integral operator

$$
\begin{aligned}
I_{\left(\beta_{m}\right), m}^{\left(\gamma_{m}\right),\left(\delta_{m}\right)} f(z) & =I_{\left(\beta_{1}, \ldots, \beta_{m}\right) ; m}^{\left(\gamma_{1}, \ldots, \gamma_{m}\right),\left(\delta_{1}, \ldots, \delta_{m}\right)} f(z) \\
& =\frac{1}{z} \int_{0}^{z} H_{m, m}^{m, 0}\left[\left.\frac{t}{z}\right|_{\left(\gamma_{k}+1-\frac{1}{\beta_{k}}, \frac{1}{\beta_{k}}\right)_{1, m}} ^{\left.\gamma_{k}+\delta_{k}+1-\frac{1}{\beta_{k}}, \frac{1}{\beta_{k}}\right)_{1, m}}\right] f(t) d t, \text { for } \sum_{1}^{m} \delta_{k}>0 \\
& =f(z), \delta_{1}=\cdots=\delta_{m}=0
\end{aligned}
$$

is said to be a multiple fractional integral operator of Riemann -Liouville type of multiorder $\delta=\left(\delta_{1}, \ldots, \delta_{m}\right)$. The definition and other related details about Fox's H-function may be found in [4, chapter 1], [5, section 8.3] (see also [9, pp. 136-137]). Following [3], let $\mathcal{H}$ denote a complex domain starlike with respect to the origin $z=0$, and $A(\mathcal{H})$ denote the space of functions analytic in $\mathcal{H}$. If $A_{\mu}(\mathcal{H})$ denote the class of functions :

$$
A_{\mu}(\mathcal{H})=\left\{f(z)=z^{\mu} \tilde{f}(z): \tilde{f}(z) \in A(\mathcal{H})\right\}, \mu \geq 0
$$

then clearly $A_{\mu}(\mathcal{H}) \subseteq A(\mathcal{H}) \subseteq A(\mathcal{H})$ for $\mu \geq 0$.

The fractional integral operator (2.7) includes various useful and important fractional integral operators as special cases. For further details, one may refer to [6] and $[9]$.

\section{Distortion Theorems}

Before, we state and prove our main results for functions belonging to general classes $J_{\delta}(n)$ and $L_{\delta}(n)$, we shall need the following results:

Lemma $1([1])$. Let the function $f(z)$ be defined by (2.4). Then $f(z)$ is in the class $J(\delta)$ if and only if

$$
\sum_{j=n+1}^{\infty}\left(\frac{j-\delta}{1-\delta}\right) a_{j} \leq 1 \quad(n \in N)
$$


Lemma 2([1]). Let the function $f(z)$ be defined by (2.4). Then $f(z)$ is in the class $L(\delta)$ if and only if

$$
\sum_{j=n+1}^{\infty}\left(\frac{j(j-\delta)}{1-\delta}\right) a_{j} \leq 1 \quad(n \in N)
$$

As noted in [10], Lemma 1 follows immediately from a result due to Silverman [10, p.110 Theorem 2] upon setting $a_{j}=0(j=2, \ldots, n)$. On the other hand, Lemma 2 is a similar consequence of another result due to Silverman [10, p.111, Corollary 2].

Lemma $3([3])$. Let $\gamma_{k}>-\frac{\mu}{\beta_{k}}-1, \delta_{k} \geq 0, \forall k=1, \ldots, m$.

Then the operator $I_{\left(\beta_{m}\right) ; m}^{\left(\gamma_{m}\right),\left(\delta_{m}\right)}$ maps the class $\mathcal{H}_{\mu}(G)$ into itself preserving the power functions $f(z)=z^{p}$ up to a constant multiplier, namely;

$$
I_{\left(\beta_{m}\right) ; m}^{\left(\gamma_{m}\right),\left(\delta_{m}\right)}\left(z^{p}\right)=\prod_{k=1}^{m}\left\{\frac{\Gamma\left(\frac{p}{\beta_{k}}+\gamma_{k}+1\right)}{\Gamma\left(\frac{p}{\beta_{k}}+\gamma_{k}+\delta_{k}+1\right)}\right\} z^{p}, \quad p \geq \mu .
$$

Our first main result is contained in the following:

Theorem 1. Let $m, h_{k} \in N$ and $\gamma_{k}, \delta_{k}, \in R$ satisfying

$$
\gamma_{k}+h_{k}+1>0 \quad \text { and } \quad \delta_{k}>0, \forall k=1, \ldots, m .
$$

If $n$ is a positive integer such that

$$
\prod_{k=1}^{m}\left(\frac{\left(1+\gamma_{k}+h_{k}(n+1)\right)_{h_{k}}}{\left(1+\gamma_{k}+\delta_{k}+h_{k}(n+1)_{h_{k}}\right.}\right) \leq 1,
$$

and $f(z)$ defined by (2.4) be in the class $J_{\delta}(n)$, then

$$
\begin{aligned}
& \left|I_{\left(h_{m}^{-1}\right) ; m}^{\left(\gamma_{m}\right),\left(\delta_{m}\right)} f(z)\right| \\
\geq & \prod_{k=1}^{m}\left\{\frac{\Gamma\left(1+h_{k}+\gamma_{k}\right)}{\Gamma\left(1+h_{k}+\gamma_{k}+\delta_{k}\right)}\right\}|z|\left[1-\frac{1-\delta}{n+1-\delta} \prod_{k=1}^{m}\left\{\frac{\left(1+\gamma_{k}+h_{k}\right)_{n h_{k}}}{\left(1+\gamma_{k}+\delta_{k}+h_{k}\right)_{n h_{k}}}\right\}|z|^{n}\right],
\end{aligned}
$$

and

$$
\begin{aligned}
& \left.\mid I_{\substack{\left(\gamma_{m}^{-1}\right),\left(\delta_{m}\right) \\
\left(\delta_{m}\right) ; m}}^{(-1} f\right) \mid \\
\leq & \prod_{k=1}^{m}\left\{\frac{\Gamma\left(1+\gamma_{k}+h_{k}\right)}{\Gamma\left(1+\gamma_{k}+\delta_{k}+h_{k}\right)}\right\}|z|\left[1+\frac{1-\delta}{n+1-\delta} \prod_{k=1}^{m}\left\{\frac{\left(1+\gamma_{k}+h_{k}\right)_{n h_{k}}}{\left(1+\gamma_{k}+\delta_{k}+h_{k}\right)_{n h_{k}}}\right\}|z|^{n}\right],
\end{aligned}
$$

for $z \in U$, and equalities are attained by the function

$$
f(z)=z-\frac{1-\delta}{n+1-\delta} z^{n+1}
$$


at certain values of $z$.

Proof. In view of formula (3.3), we have

$$
I_{\left(h_{m}^{-1}\right) ; m}^{\left(\gamma_{m}\right),\left(\delta_{m}\right)} f(z)=\prod_{k=1}^{m}\left\{\frac{\Gamma\left(1+\gamma_{k}+h_{k}\right)}{\Gamma\left(1+\gamma_{k}+\delta_{k}+h_{k}\right)}\right\} z-\sum_{j=n+1}^{\infty} \prod_{k=1}^{m}\left\{\frac{\Gamma\left(1+\gamma_{k}+h_{k} j\right)}{\Gamma\left(1+\gamma_{k}+\delta_{k}+h_{k} j\right)}\right\} a_{j} z^{j}
$$

Define a function $\phi(z)$ by

$$
\phi(z)=\prod_{k=1}^{m}\left\{\frac{\Gamma\left(1+\gamma_{k}+\delta_{k}+h_{k}\right)}{\Gamma\left(1+\gamma_{k}+h_{k}\right)}\right\} I_{\left(h_{m}^{-1}\right) ; m}^{\left(\gamma_{m}\right),\left(\delta_{m}\right)} f(z)=z-\sum_{j=n+1}^{\infty} \Psi(j) a_{j} z^{j}
$$

where

$$
\Psi(j)=\prod_{k=1}^{m} \frac{\left(1+\gamma_{k}+h_{k}\right)_{h_{k}(j-1)}}{\left(1+\gamma_{k}+\delta_{k}+h_{k}\right)_{h_{k}(j-1)}} \quad(j=n+1, n+2, \ldots)
$$

which is observed to be non-increasing for integers $j \geq n+1$, by virtue of the formula

$$
(a)_{m j}=m^{m j}\left(\frac{a}{m}\right)_{j}\left(\frac{a+1}{m}\right)_{j} \cdots\left(\frac{a+m-1}{m}\right)_{j}(m \in N)
$$

and the conditions (3.4) and (3.5), implying thereby that

$$
0<\Psi(j) \leq \Psi(n+1)=\prod_{k=1}^{m}\left\{\frac{\left(1+\gamma_{k}+h_{k}\right)_{n h_{k}}}{\left(1+\gamma_{k}+\delta_{k}+h_{k}\right)_{n h_{k}}}\right\}
$$

From Lemma 1, we also have

$$
\sum_{j=i n+1}^{\infty} a_{j} \leq \frac{1-\delta}{n+1-\delta}
$$

and hence (3.10), (3.13) and (3.14) yield

$$
|\phi(z)| \geq|z|-\Psi(n+1)|z|^{n+1} \sum_{j=n+1}^{\infty} a_{j} \geq|z|-\frac{1-\delta}{n+1-\delta} \Psi(n+1)|z|^{n+1} .
$$

This implies the assertion (3.6) of Theorem 1. The assertion (3.7) can be proved similarly. It can be easily verified that the equalities in (3.6) and (3.7) hold when $z=|z|$.

By applying Lemma 2(instead of Lemma 1 ) to the function $f(z)$ belonging to the class $L_{\delta}(n)$, we can prove the following:

Theorem 2. Under the assumptions (3.4) and (3.5) of Theorem 1, let the function $f(z)$ defined by $(2.4)$ be in the class $L_{\delta}(n)$. Then

$$
\begin{aligned}
\mid I_{\left(\begin{array}{l}
\left.h_{m}^{-1}\right) ; m \\
\left(\gamma_{m}\right),\left(\delta_{m}\right)
\end{array} f(z) \mid\right.} & \geq \prod_{k=1}^{m}\left\{\frac{\Gamma\left(1+\gamma_{k}+h_{k}\right)}{\Gamma\left(1+\gamma_{k}+\delta_{k}+h_{k}\right)}\right\}|z| \\
\times & {\left[1-\frac{(1-\delta)}{(n+1)(n+1-\delta)} \prod_{k=1}^{m}\left\{\frac{\left(1+\gamma_{k}+h_{k}\right)_{n h_{k}}}{\left(1+\gamma_{k}+\delta_{k}+h_{k}\right)_{n h_{k}}}\right\}|z|^{n}\right], }
\end{aligned}
$$


and

$$
\begin{aligned}
\left|I_{\left(h_{m}^{-1}\right) ; m}^{\left(\gamma_{m}\right),\left(\delta_{m}\right)} f(z)\right| & \leq \prod_{k=1}^{m}\left\{\frac{\Gamma\left(1+\gamma_{k}+h_{k}\right)}{\Gamma\left(1+\gamma_{k}+\delta_{k}+h_{k}\right)}\right\}|z| \\
& \times\left[1+\frac{(1-\delta)}{(n+1)(n+1-\delta)} \prod_{k=1}^{m}\left\{\frac{\left(1+\gamma_{k}+h_{k}\right)_{n h_{k}}}{\left(1+\gamma_{k}+\delta_{k}+h_{k}\right)_{n h_{k}}}\right\}|z|^{n}\right] .
\end{aligned}
$$

Equalities in (3.15) and (3.16) are attained by the function

$$
f(z)=z-\frac{1-\delta}{(n+1)(n+1-\delta)} z^{n+1}
$$

at certain values of $z$.

\section{Interesting deductoins}

In this section we deduce some results giving the distortion inequalities for fractional integral operators which are special cases of (2.7).

To this end, we put

$m=1, h_{1}=h, \gamma_{1}=\eta, \delta_{1}=\alpha$ in (2.7), so that from [6,p.223, Eqn. (3.7)]:

$$
I_{h^{-1} ; 1}^{\eta, \alpha} f(z) \rightarrow h E_{o, z ; h^{-1}}^{\alpha, \eta} f(z)
$$

With the above specialization in Theorems 1 and 2 , we arrive at the following results involving the Erdélyi- Kober fractional integrals:

Corollary 1. Let the function $f(z)$ defined by (2.4) be in the class $J_{\delta}(n)$. Then under conditions easily obtainable from (3.4) and (3.5):

$$
\left|E_{o, z ; h^{-1}}^{\alpha, \eta} f(z)\right| \geq\left\{\frac{\Gamma(1+\eta+h)}{h \Gamma(1+\eta+\alpha+h)}\right\}|z|\left\{1-\frac{(1-\delta)(1+\eta+h)_{n h}}{(n+1-\delta)(1+\eta+\alpha+h)_{n h}}|z|^{n}\right\}
$$

and

$$
\left|E_{o, z ; h^{-1}}^{\alpha, \eta} f(z)\right| \leq\left\{\frac{\Gamma(1+\eta+h)}{h \Gamma(1+\eta+\alpha+h)}\right\}|z|\left\{1+\frac{(1-\delta)(1+\eta+h)_{n h}}{(n+1-\delta)(1+\eta+\alpha+h)_{n h}}|z|^{n}\right\}
$$

for $z \in U$.

Equalities in (4.2) and (4.3) are attained by function given by (3.8).

Corollary 2. Let the function $f(z)$ defined by (2.4) be in the class $L_{\delta}(n)$. Then under conditions easily obtainable from (3.4) and (3.5):

$$
\begin{aligned}
\left|E_{o, z ; h^{-1}}^{\alpha, \eta} f(z)\right| \geq & \left\{\frac{\Gamma(1+\eta+h)}{h \Gamma(1+\eta+\alpha+h)}\right\}|z| \\
& \times\left\{1-\frac{(1-\delta)(1+\eta+h)_{n h}}{(n+1-\delta)(n+1)(1+\eta+\alpha+h)_{n h}}|z|^{n}\right\}
\end{aligned}
$$


and

$$
\left|E_{o, z ; h^{-1}}^{\alpha, \eta} f(z)\right| \leq\left\{\frac{\Gamma(1+\eta+h)}{h \Gamma(1+\eta+\alpha+h)}\right\}|z|\left\{1+\frac{(1-\delta)(1+\eta+h)_{n h}}{(n+1-\delta)(n+1)(1+\eta+\alpha+h)_{n h}}|z|^{n}\right\}
$$

for $z \in U$.

Equalities in (4.4) and (4.5) are attained by the function given by (3.17) at certain values of $z$.

Next we set

$$
\begin{aligned}
& m=2, \gamma_{1}=\eta-\beta, \gamma_{2}=0, \delta_{1}=-\eta, \delta_{2}=\alpha+\eta, \\
& h_{1}=h_{2}=1, \text { in }(2.7), \text { so that from }[6, \text { p.223, Eqn. (3.9)]: }
\end{aligned}
$$

$$
I_{1,1 ; 2}^{(\eta-\beta, o),(-\eta, \alpha+\eta)} f(z) \rightarrow z^{\beta} I_{o, z}^{\alpha, \beta, \eta} f(z) .
$$

Theorems 1 and 2 with above substitutions yield the following results [12, Theorems 1 and 2, pp. 416-419] involving the Saigo fractional integrals [6]:

Corollary 3. Let the function $f(z)$ defined by (2.4) be in the class $J_{\delta}(n)$. Then under conditions easily obtainable from (3.4) and (3.5):

$$
\begin{aligned}
\left|I_{o, z}^{\alpha, \beta} f(z)\right| \geq & \left\{\frac{\Gamma(2-\beta+\eta)}{\Gamma(2-\beta) \Gamma(2+\alpha+\eta)}\right\}|z|^{1-\beta} \\
& \times\left\{1-\frac{(1-\delta)(-\beta+\eta+2)_{n}(n+1) !}{(n+1-\delta)(-\beta+2)_{n}(\alpha+\eta+2)_{n}}|z|^{n}\right\},
\end{aligned}
$$

and

$$
\begin{aligned}
\left|I_{o, z}^{\alpha, \beta, \eta} f(z)\right| \leq & \left\{\frac{\Gamma(2-\beta+\eta)}{\Gamma(2-\beta) \Gamma(2+\alpha+\eta)}\right\}|z|^{1-\beta} \\
& \times\left\{1+\frac{(1-\delta)(-\beta+\eta+2)_{n}(n+1) !}{(n+1-\delta)(-\beta+2)_{n}(\alpha+\eta+2)_{n}}|z|^{n}\right\},
\end{aligned}
$$

for $z \in U$ if $\beta \leq 1$ and $z \in U-\{0\}$ if $\beta>1$. Equalities in (4.7) and (4.8) are attained by the function given by (3.8) at certain values of $z$, where $\beta$ is assumed to be a rational number for the case (4.8).

Corollary 4. Let the function $f(z)$ defined by (2.4) be in the class $L_{\delta}(n)$. Then under conditions easily obtainable from (3.4) and (3.5):

$$
\begin{aligned}
\left|I_{o, z}^{\alpha, \beta, \eta} f(z)\right| \geq & \left(\frac{\Gamma(2-\beta+\eta)}{\Gamma(2-\beta) \Gamma(2+\alpha+\eta)}\right)|z|^{1-\beta} \\
& \times\left\{1-\frac{(1-\delta)(-\beta+\eta+2)_{n} n !}{(n+1-\delta)(-\beta+2)_{n}(\alpha+\eta+2)_{n}}|z|^{n}\right\}
\end{aligned}
$$


and

$$
\begin{aligned}
\left|I_{o, z}^{\alpha, \beta, \eta} f(z)\right| \leq & \left(\frac{\Gamma(2-\beta+\eta)}{\Gamma(2-\beta) \Gamma(2+\alpha+\eta)}\right)|z|^{1-\beta} \\
& \times\left\{1+\left(\frac{(1-\delta)(-\beta+\eta+2)_{n} n !}{(n+1-\delta)(-\beta+2)_{n}(\alpha+\eta+2)_{n}}\right)|z|^{n}\right\} .
\end{aligned}
$$

for $z \in U$ for $\beta \leq 1$ and $z \in U-\{0\}$ for $\beta>1$. Equalities in (4.9) and (4.10) are attained by the function given by (3.17).

We conclude this paper by remarking that various other classes of distortion inequalities can be deduced from the main results (Theorems 1 and 2) in view of the fact (see [9]) that several know forms of fractional integral operators emerge from the general fractional integral operator (2.7). The deductions of these obvious consequences being quite straightforward, further details are hence omitted.

\section{Acknowledgement}

The work of the first author was supported by National Board for Higher Mathematics (DAE), Govt. of India Under Grant No.26/6/93-G.

\section{References}

[1] S. K. Chatterjea, "On Starlike functions," J. Pure Math., 1(1981), 23-26.

[2] S. L. Kalla and V. S. Kiryakova, "An H-function generalized fractional calculus based upon compositions of Erdélyi-Kober operators in $L_{p}$, , Math. Japon, 35(1990), 1151-1171.

[3] V. S. Kiryakova, "Generalized $H_{m, m}^{m, 0}$-function fractional integration operators in some classed of analytic functions," Math. Vesnik, 40(1988), 259-266.

[4] A. M. Mathai and R. K. Saxena, The H-function with Applications in Statistics and Other Disciplines, Halsted Press, New York - London - Sydney - Toronto, 1978.

[5] A. P. Prudnikov, Ju. A. Brychkov and O. I. Marichev, Integrals and Series, Vol.3, More Special Functions, Gordon and Breach, New York - Philadelphia - London - Paris - Montreux - Tokyo -Melbourne, 1990.

[6] R. K. Raina and M. Saigo, "A note on fractional calculus operators involving Fox's H-function on space $F_{p, \mu}$ " in Recent Advances in Fractional Calculus, Edited by R. N. Kalia, Global Publ, Sauk Rapids, 219-229, 1993.

[7] M. S. Robertson, "On the theory of univalent functions," Ann. of Math., 37(1936), 374-408.

[8] M. Saigo, "A remark on integral operators involving the Gauss hypergeometric functions," Math. Rep. College General Ed. Kyushu Univ., 11(1978), 135-143.

[9] M. Saigo, R. K. Raina and A. A. Kilbas, "On generalized fractional calculus operators and their compositions with axisymmetric differential operators of the potential theory on spaces $F_{p, \mu}$ and $F_{p, \mu}^{\prime} . "$ Fukuoka Univ. Sci. Rep., 23(2)(1993), 133-154.

[10] H. Silverman, "Univalent functions with negative coefficients," Proc. Amer. Math. Soc., 51(1975), 119-116.

[11] H. M Srivastava, S. Owa and S. K. Chatterjea, "A note on certain classes of starlike functions," Rend. Sem. Mat. Univ. Padova, 77(1987), 115-124. 
[12] H. M. Srivastava, M. Saigo and S. Owa, "A class of distortion theorems involving certain operators of fractional calculus," J. Math. Anal. Appl., 131(2)(1988), 412-420.

Department of Mathematics, C. T. A. E., Campus, Udaipur UDAIPUR -313 001 Rajasthan, INDIA. Department of Mathematics, College of Science, Sukhadia University, UDAIPUR -313 001, Rajasthan, INDIA. 